\title{
Der Arbeitskreis Polarlehrer
}

\section{Rainer Lehmann}

Arbeitskreis Polarlehrer, Europa-Universität Flensburg, Physische Geographie, Auf dem Campus 1, 24943 Flensburg, Deutschland

Correspondence: Rainer Lehmann (ak-polarlehrer@ polarforschung.de, rainer.lehmann@ uni-flensburg.de)

Published: 20 May 2021

\section{Rückblick des AK Polarlehrer}

Der Arbeitskreis Polarlehrer ist ein Netzwerk aus Wissenschaftler*innen und Lehrkräften, die gemeinsam die Rahmenbedingungen für eine lebendige Wissenschaftskommunikation geschaffen haben. Diese innovative Form der $\mathrm{Zu}$ sammenarbeit zwischen Wissenschaft und Schulen hat u.a. im Internationalen Polarjahr 2007/08 die Initiative zum Projekt Coolen Klassen ermöglicht, welches 5 Jahre von der Robert Bosch Stiftung gefördert wurde (Lehmann und Huch, 2008). In diesem bundesweiten, interdisziplinären Projekt stand die Vermittlung von Polarwissenschaften in die Schulen im Fokus. Wissenschaftler*innen und Lehrkräfte als Multiplikatoren haben sich mit ihren Lerngruppen in unterschiedlichen, individuell geprägten Projekten eingebracht.

Aus dem Projekt Coole Klassen ist 2008 der Arbeitskreis Polarlehrer in der Deutschen Gesellschaft für Polarforschung (DGP) hervorgegangen (https: //www.polarforschung.de/arbeitskreise/ak-polarlehrer/,

letzter Zugriff: 17. Februar 2021). Er bietet seit 14 Jahren neben einem wichtigen Netzwerk auch Fortbildungen, Newsletter, Publikationen, die Vermittlung von Kontakten zwischen Wissenschaftlern und Lehrkräften und die Beteiligung von Lehrkräften an Polarexpeditionen (Pound et al., 2019). Der Arbeitskreis ist bundesweit aktiv und vermittelt wissenschaftliche Fragestellungen, Methoden bzw. Arbeitsweisen und Ergebnisse der Polarforschung an junge Menschen und ist daher auf dem Feld der Wissenschaftskommunikation sehr wertvoll. Er übersetzt aktuelle Polarforschung in adressatengerechte und dialogorientierte Materialien (Pulz und Roser, 2011; Theisen und Visbeck, 2011; Knickmeier et al., 2011; Lehmann und May, 2013; Kallfelz und Lehmann, 2015; Krüger, 2020, 2021; Lehmann und Wüthrich, 2020; Friedrich Verlag, 2015), die im Unterricht und in Projekten an Schulen und in Museen genutzt werden. Viele Angebote sind auch online verfügbar (z.B. https://mosaic-expedition.org/bildung/, letzter Zugriff: 17. Februar 2021). Der Arbeitskreis bietet bei seinen Veranstaltungen wie beispielsweise regelmäßigen Arbeitskreis-Treffen, den Internationalen Polartagungen der DGP oder Workshops Orte für den Austausch von Wissenschaft und Schule (Lehmann und Kallfelz, 2019).

Die Stetigkeit in der Arbeit der Lehrkräfte ist für die Effizienz des Kreises unabdingbar und wird beispielsweise durch die Informationsverbreitung im Newsletter und die jährlichen Arbeitskreis-Treffen gewährleistet. Eine Herausforderung ist seit seinem Bestehen die Erreichbarkeit der Lehrkräfte, da es keinen zentralen Verteiler für Lehrkräfte in Deutschland gibt. Dieses Problem wird teilweise durch die Kooperation mit bundesweiten Fachverbänden wie den Verband deutscher Schulgeographen (VDSG) gelöst. Die deutsche Gesellschaft für Polarforschung sowie das SPP Antarktisforschung unterstützen den Arbeitskreis dankenswerterweise bei Arbeitskreis-Treffen und Tagungen. Für die Zukunft ist eine breite finanzielle Basis wünschenswert, um beispielsweise Lehrkräfte bei Fortbildungen durch den AK Polarlehrer oder (Dienst-) Reisen im Zusammenhang mit der Netzwerkarbeit zu fördern.

Enge Kooperationspartner sind das Alfred-WegenerInstitut Helmholtz-Zentrum für Polar- und Meeresforschung (https://www.awi.de/, letzter Zugriff: 17. Februar 2021, https://mosaic-expedition.org/, letzter Zugriff: 17. Februar 2021) und das DFG-Schwerpunktprogramm 1158 - Antarktisforschung (https://www.spp-antarktisforschung.de/, letzter Zugriff: 17. Februar 2021) sowie mehrere Universitäten. Förderlich ist die Vernetzung und der Austausch des Arbeitskreises gerade mit jungen Wissenschaftler*innen der Association of Polar Early Career Scientists (APECS, https: //www.apecs.is/, letzter Zugriff: 17. Februar 2021), die noch eng mit der jungen Generation verbunden sind (Lehmann 
und Rudolph, 2020). International kooperiert der Arbeitskreis (Polar Educators Germany) mit Polar Educators International (PEI, https://polareducator.org/, letzter Zugriff: 17. Februar 2021), eine multinationale Vereinigung, die 2012 nach dem Vorbild des Arbeitskreises gegründet wurde (May et al., 2013; Huffman et al., 2015).

\section{Interview mit dem Leiter des AK Polarlehrer R. Lehmann}

Authors: Rainer Lehmann, Tim Carlsen

T. Carlsen (APECS Germany): Kurz zu Ihrer Person und Ihrem beruflichen Werdegang. Wollten Sie schon immer Polarforscher werden? Wann und warum haben Sie sich für die Polarforschung entschieden?

R. Lehmann: Im Alter von 14 Jahren habe ich mir eine große Karte von Alaska im Zimmer aufgehängt und das Iditarod-Hundeschlittenrennen mitverfolgt, so gut, wie es damals eben ging. In diese Zeit fiel auch meine erste Reise in das norwegische Gebirge. Seitdem begeistere ich mich für den Norden. Die Polargebiete übten schon eine große Anziehungskraft auf mich aus, waren aber im Gegensatz zu Norwegen, Finnland oder Alaska noch unerreichbar.

Während des Studiums der Geographie, Geologie und Biologie nahm ich mir ein Freisemester und lebte ein halbes Jahr in Alaska, im Yukon und den Nordwestterritorien. Dabei nahm ich auch an der IV Int. Conference on Permafrost in Fairbanks teil. Die erste wissenschaftliche, polare Expedition unter der Leitung von Prof. Dr. Lorenz King führte mich als Geomorphologe in die kanadische Hocharktis nach Axel Heiberg und Ellesmere Island. Die Faszination dieser Geoökosysteme nahm mit dieser Expedition noch weiter zu. Seitdem bin ich auch Mitglied in der DGP.

Meine Biographie führte mich nach der Dissertation (die konträr zu den Polargebieten im Mittelmeerraum durchgeführt wurde) über den Umweg der freien Wirtschaft in die Schule, um Schüler*innen der Oberstufe in Geographie und Biologie zu unterrichten. Ein wichtiger Schwerpunkt meines Unterrichts lag hier immer beim Thema Polargebiete, zu dem auch viele, auch internationale Projekte mit Schüler*innen durchgeführt wurden.

Das Internationale Polarjahr 2007/08 führte mich mit Dr. Franz Tessensohn zusammen, und wir initiierten das Schulprojekt Coole Klassen, aus dem der Arbeitskreis Polarlehrer hervorgegangen ist.

APECS Germany: Warum setzen Sie sich für das Thema Ihres AK ein? Was macht dieses Forschungsfeld für Sie so besonders, dass Sie dafür so viel Zeit und Einsatz bringen?

R. Lehmann: Die Ausgangssituation stellt sich sehr konträr dar: Während die Polargebiete für die junge Generation ein spannendes Themenfeld darstellen, werden sie im Gegensatz zu den anderen Gebieten der Erde in den Schulen zu wenig unterrichtet. Junge Menschen kennen beispielsweise oft grundsätzliche Charakteristika und Unterschiede zwischen Arktis und Antarktis nicht, von Zusammenhängen in diesen Gebieten ganz zu schweigen (vgl. Conrad, 2012). Das ist in den Curricula der Schulfächer Geographie und Biologie besonders auffällig. Gerade in der Gegenwart, in der der Wandel in den Polargebieten am dramatischsten ist, sollen junge Menschen ein Verständnis für diese Gebiete, den Lebensraum und die Menschen dort entwickeln. Nur dadurch, nur durch das Wissen der Prozesse, Regler, Speicher und deren Rückkopplungen werden junge Menschen in der Lage sein, regionale und globale Zusammenhänge zu erkennen, sie zu bewerten und Schlussfolgerungen daraus zu ziehen. Die Gesellschaft insgesamt hat eine Verantwortung gegenüber dem Nachwuchs, das zu ermöglichen. Die DGP bietet eine Plattform, Wissenschaft und Schule in diesem Sinn zusammen zu bringen.

Zudem kann die Begeisterung von Schüler*innen für die Pole der Erde genutzt werden, um die in den Curricula verankerten, grundsätzlichen Systemzusammenhänge anhand spannender Beispiele aus den Polargebieten zu vermitteln. Den jungen Menschen wird durch die emotionale Basis gegenüber den kalten, unzugänglichen Regionen der Erde mithilfe polarer Themen erleichtert, auch komplexe Sachverhalte zu erkennen und Kompetenzen wie Erkenntnisgewinnung, Bewertung oder Kommunikation sowie inhaltsbezogene Kompetenzen zu entwickeln.

Wenn von Forschungsfeld die Rede ist: Es wäre sehr wünschenswert, dass unsere Arbeit wissenschaftlich evaluiert und begleitet werden würde, um ihre Effizienz zu erhöhen.

APECS Germany: Was gefällt Ihnen an Ihren Aufgaben als AK Leiter ganz besonders? Hat sich dadurch Ihre Sicht auf etwas geändert?

R. Lehmann: Ich beschäftige mich mit den Themen, die mir besonders am Herzen liegen. Durch meinen wissenschaftlichen Hintergrund und die Unterrichtserfahrungen mit Schüler*innen befinde ich mich an der Schnittstelle zwischen Wissenschaft und Schule. Dadurch sind mir beide Seiten zugänglich und ihre Sichtweisen verständlich.

Die Arbeit für den Arbeitskreis ermöglicht eine enge $\mathrm{Zu}$ sammenarbeit mit wissenschaftlichen Forschungseinrichtungen wie z.B. dem AWI und Universitäten sowie mit Kollegen aus Schulen und Museen in ganz Deutschland. Dadurch entstehen lebendige Kontakte zu Menschen, die sich für die gleichen Themen begeistern, mit denen man zusammenarbeiten und sich austauschen kann, von denen man lernen kann. Wesentlich ist der Blick ,über den Tellerrand“ Schule hinaus.

Die Sichtweise auf die Möglichkeiten des Arbeitskreises ändert und entwickelt sich natürlich, je mehr und je länger man sich damit beschäftigt. Was ursprünglich aus meiner Begeisterung für die Polarregionen und der Unterrichtserfahrung an der Schule als Projekt entstand, ist inzwischen mehr der Wissenschaftskommunikation zuzuordnen. Nicht im herkömmlichen Sinn, sondern als neuer Ansatz, wie aktuelle, wissenschaftliche Fragestellungen, Arbeitsmethoden und Er- 
gebnisse (zu den Polargebieten) in die Klassenzimmer transferiert werden können.

APECS Germany: Wenn Sie in die Zukunft schauen, was würden Sie gerne im Feld Ihres Arbeitskreises in fünf Jahren herausgefunden haben? Was ist in Ihren Augen die dringendste Frage im Feld Ihres Arbeitskreises?

R. Lehmann: Eine Erhebung der Breitenwirkung der Tätigkeiten des Arbeitskreises, insbesondere bei unterschiedlichen Kommunikationsprojekten, würde uns helfen, bestimmte Ansätze wie z.B. Fortbildungen oder die Entwicklung von Unterrichtsmaterialien gezielt zu fördern. Auf den Punkt gebracht: Welche Maßnahmen führen zu einer Weiterentwicklung der Wissenschaftskommunikation im Schulbereich, und wie können sie gestärkt, gefördert oder initiiert werden. Hürden und Herausforderungen sind die 16 Bundesländer sowie keine oder nur sehr begrenzte finanzielle Mittel.

Dringende Fragen sind: Wie begeistern wir die Wissenschaftspolitik für eine kontinuierliche Kooperation zwischen Wissenschaft und Schule? Wie kann die Arbeit des Arbeitskreises kontinuierlich und auch finanziell unterstützt werden? Wie kann die Erreichbarkeit der Lehrkräfte gesteigert werden?

APECS Germany: Was darf für Sie bei der (Feld-)arbeit nie fehlen?

R. Lehmann: Feldbuch, Bleistift, Karten, Probenbeutel, Fernglas, Kamera, Sitzkissen, und Schokolade.

APECS Germany: Was war Ihre letzte größere Expedition und nutzen Sie solche Erfahrungen für den eigenen Unterricht?

R. Lehmann: Von September bis Dezember 2007 war ich auf der McMurdo Forschungsstation in der Antarktis. Als Teil des internationalen Bohrprojekts ANDRILL analysierten wir dort Sedimentbohrkerne aus dem Rossmeer. Gerade die Materialien aus den Expeditionen kann ich direkt im Unterricht verwenden: Fotos, Kartenmaterial, selbst erstellte Aufgabenblätter, aber zum Beispiel auch selbst gesammelte Gesteinsproben.

Expeditionen sind nicht nur sehr attraktiv für Lehrkräfte, man wird auch für die Schüler authentischer. Deshalb würde ich mir wünschen, dass Expeditionsteilnahmen für Lehrende (wie im Rahmen des Projekts Coole Klassen in der Vergangenheit bereits geschehen) wieder regelmäßiger angeboten werden.

APECS Germany: Was war Ihre einprägsamste (lustig/beängstigend/seltsam) Erfahrung in der Polarforschung?

R. Lehmann: Das Schönste ist für mich die Natur, die man erforscht. Am einprägsamsten ist das Gefühl der Unberührtheit, der Weite und Wildheit des Naturraumes, das vor allem erlebbar ist, wenn man alleine oder in kleinen Gruppen zu Fuß im Gelände unterwegs ist.

Die Naturgewalten sind mir an einem Tag am Thompson Gletscher auf Axel Heiberg Island besonders bewusst geworden. Durch starke Sonneneinstrahlung nach einem Wetterumschwung verwandelten sich Rinnsale vor dem Gletscher in reißende Ströme. Das zeigt auch wie wichtig Erfahrung ist, um bei solchen Expeditionen sicher wieder zurückzukommen.

Datenverfügbarkeit. Für diesen Artikel wurden keine Datensätze genutzt.

Interessenkonflikt. Die Autor*innen erklären, dass kein Interessenkonflikt besteht.

Begutachtung. This paper was edited by Donovan Dennis.

\section{Literatur}

Conrad, D.: Schülervorstellungen zur eisigen Welt der Polargebiete. Ergebnisse einer explorativ angelegten Studie, GuiD 3/12, S. 105-127, 2012.

Friedrich Verlag (Hrsg.): Polarforschung hautnah - Biologie 5-10, Nr. 12/2015, 48 Seiten, Materialpaket mit Experimentierwerkstatt Polarforschung, 2015.

Huffman, L., Xavier, J., Beck, I. und Lehmann, R.: Education Meets Science: Bringing polar research into the classroom (1-4 April 2015, Hannover, Germany), The Polar Journal, Vol. 5, 2, Conference Reports, 472-473, 2015.

Kallfelz, M. und Lehmann, R.: Kieselalgen im Dienst der Klimaforschung, Biologie 5-10, Nr. 12/2015, Polarforschung hautnah, 28-31, Materialpaket, 2015.

Knickmeier, K., Lohse, C. und Sommer, U.: Biologische Ozeanographie, in: Abenteuer Weltmeere. Reihe Naturwissenschaften Biologie - Chemie - Physik, Herausgeber: Dietrich, V., Erb, R. und Kleesattel, W., Cornelsen Verlag, 64 Seiten, ISBN 978-3-06010828-2, 2011.

Krüger, F.: Expedition in die Arktis: Ein Tag auf dem Eis, Geographie heute, 350, 46-49, 2020.

Krüger, F.: Expedition in die Arktis, Biologie 5-10, 33, Klimawandel, im Druck, 2021.

Lehmann, R. und Huch, M.: Coole Klassen - Schulprojekte im IPY, Das Internationale Polarjahr 2007/08, Folge 8, Polarforschung, 77, 37-38, 2008.

Lehmann, R. und Kallfelz, M.: Die Polarlehrer - Coole Klassen an den Polen der Erde, Biologie in unserer Zeit, 49, 277-281, 2019.

Lehmann, R. und May, I.: Polargebiete, In der Reihe: Themenhefte Erdkunde - Landschaftszonen der Erde entdecken, Verlag an der Ruhr, 48 Seiten, 2013.

Lehmann, R. und Rudolph, E.: Polar Educators Germany, ECO Magazine Polar Special Issue, 124-125, available at: http://digital.ecomagazine.com/publication/?i=674747\&ver= html5\&p=124 (letzter Zugriff: 18. Februar 2021), 2020.

Lehmann, R. und Wüthrich, C.: Seevögel und die Vogelklifftundra, Biologie 5-10, Nr. 32/2020, Die Welt der Vögel, 36-39, Materialpaket, 2020.

May, I., May, K. und Lehmann, R.: Polarwissenschaften in der Schule - Internationaler Workshop für Lehrer und Wissenschaftler, Polarforschung, 82, 151-152, 2013.

Pound, K., Huffman, L., Hubbard, J., Cattadori, M., Dahlman, L., Dooley, J., Frisch-Gleason, R., Lehmann, R. und Trummel, 
B.: ANDRILL ARISE: A model for team-based field research immersion for educators, Polar Record, 55, 4, 251-273, https://doi.org/10.1017/S0032247419000056, 2019.

Pulz, H. und Roser, H.: Klimawandel und Polargebiete. Ein Unterrichtsvorschlag für Klasse 10, Herausgeber: Landesinstitut für Schulentwicklung Baden-Württemberg: Naturwissenschaft und Technik, NwT 9, Allgemein bildende Schulen, Gymnasium, 84 Seiten, 2011.
Theisen, S. und Visbeck, M.: Physikalische Ozeanographie, in: Abenteuer Weltmeere. Reihe Naturwissenschaften Biologie Chemie - Physik, Herausgeber: Dietrich, V., Erb, R. und Kleesattel, W., Cornelsen Verlag, 64 Seiten, ISBN 978-3-06-010828-2, 2011. 